\title{
Waldmann-Snider Collision Integrals for Mixtures of Polyatomic Gases Exact and Approximate Relations
}

\author{
W. E. Köhler \\ Institut für Theoretische Physik, Universität Erlangen-Nürnberg \\ and G. W. 't Hooft* \\ Huygens-Laboratorium, Rijksuniversiteit Leiden, The Netherlands \\ Z. Naturforsch. 34a, 1255-1268 (1979); received August 30, 1979
}

\begin{abstract}
Collision brackets of the linearized Waldmann-Snider collision superoperator are studied for mixtures of polyatomic gases. Properties following from symmetries of molecular interaction and collisional conservation laws are discussed. For a complete system of expansion tensors bracket symbol expressions for effective cross sections are given which generalize the Chapman-Cowling $\Omega$-integrals. Exact relations between effective cross sections are derived. Approximate relations which hold for molecules with small nonsphericity and elastic scattering are also given.
\end{abstract}

\section{Introduction}

Transport phenomena with mixtures of polyatomic gases, especially diatomic molecule-noble gas mixtures, in the presence of external fields (Senftleben-Beenakker effects) have been thoroughly investigated during the last decade [1-4a]. Also optical studies as depolarized Rayleigh scattering [5] and flow birefringence [6] have been performed for mixtures of optically anisotropic molecules with noble gas atoms. The appropriate theoretical treatment of these effects, based on the WaldmannSnider kinetic equation [7], started with a paper by Raum and Köhler [8] in which the relevant moment equations were derived. This system of moment equations was used to treat heat conduction [9] and thermal diffusion [10] in a magnetic field as well as flow birefringence [11] and diffusio birefringence [12] for mixtures of linear molecules with noble gas atoms. The corresponding Chapman-Enskog treatment of all transport phenomena for gaseous mixtures in a magnetic field has recently been given by Eggermont, Vestner and Knaap [13]. The Chapman-Enskog method was extended to cover also Burnett effects with polyatomics like the viscomagnetic diffusion flux [14].

A common feature in the theory of these effects is that their magnitudes are essentially determined by

* Present address: Natuurkundig Laboratorium, N. V. Philips, Eindhoven, The Netherlands

Reprint requests to Dr. W. E. Köhler, Institut für Theoretische Physik, Universität Erlangen-Nürnberg, Glückstraße 6, D-8520 Erlangen. matrix elements (collision integrals) of the Waldmann-Snider collision operator. The collision integrals can in principle be evaluated if the binary scattering amplitude is known. The scattering matrix is linked with the nonspherical molecular interaction [15] via the well known scattering integral equation and is thus difficult to obtain. In a number of papers $[16-19]$ the approximate calculation of Waldmann-Snider collision integrals for pure gases of molecules with small nonsphericity has been discussed.

In the moment also exact calculations based on the $S$-matrix formalism are underway [20].

Rather than to evaluate collision integrals for mixtures explicitly, here like in Refs. [21, 22] general expressions for the pertaining effective cross sections and exact interrelations between them (i.e. not depending on the specific form of the intermolecular potential) are discussed. This is nevertheless helpful and important since first of all for most molecules reliable scattering calculations don't yet exist, and secondly such interrelations between cross sections serve to reduce the number of unknown parameters to be determined from experiments. Further more, the general expressions already often show which kind of molecular collision processes (e.g. elastic or inelastic ones) contribute to a certain cross section.

In the present paper first a review of general properties of collision integrals linked with the symmetries of molecular interaction and conservation laws is given. Then scalar quantities, the

0340-4811 / $79 / 1100-1255 \$ 01.00 / 0$. - Please order a reprint rather than making your own copy. 
effective cross sections, are defined. They represent the parameters to be determined in experiments. A set of expansion tensors sufficient to describe all transport and relaxation phenomena in mixtures measured up to now is introduced and the effective cross sections are (after the integration over the c.m. velocity) written in terms of lucid bracket symbols. At this stage already, interesting nontrivial connections between various effective cross sections are obtained. Finally, some approximate relations which hold in the case of small nonsphericity of the interaction, are derived and a number of effective cross sections is - in spherical approximation [22] - given in terms of ChapmanCowling [23] collision integrals.

\section{General Properties of the Collision Integrals}

\section{Definitions}

Consider a mixture of polyatomic gases in a state close to thermal equilibrium. The distribution operator for the molecules of species " $k$ " (which is a density operator with respect to internal molecular rotational states) is written as

$f_{k}\left(t, \boldsymbol{x}, \boldsymbol{W}_{k}, \boldsymbol{J}_{k}\right)=f_{k}^{(0)}\left(1+\Phi_{k}\left(t, \boldsymbol{x}, \boldsymbol{W}_{k}, \boldsymbol{J}_{k}\right)\right)$.

Here, $t$ is the time, $\boldsymbol{x}$ is the position, $\boldsymbol{W}_{k}=$ $\left(m_{k} / 2 k_{\mathrm{B}} T\right)^{1 / 2}\left(\boldsymbol{c}_{k}-\boldsymbol{v}\right)$ is the dimensionless peculiar molecular velocity and $\boldsymbol{J}_{k}$ is the rotational angular momentum in units of $\hbar$. The equilibrium distribution function

$$
\begin{aligned}
f_{k}^{(0)}= & n_{k}\left(m_{k} / 2 \pi k_{\mathrm{B}} T\right)^{3 / 2}\left(Z_{k}^{\text {rot }}\right)^{-1} \\
& \cdot \exp \left(-W_{k}^{2}-\frac{H_{k}^{\text {rot }}}{k_{\mathrm{B}} T}\right)
\end{aligned}
$$

is either a local equilibrium distribution (which is used for a Chapman-Enskog treatment of the linearized Waldmann-Snider equation [13]; $\boldsymbol{v}$ then being the mean mass velocity of the gas) or a global equilibrium distribution (for a moment method treatment [8]; $\boldsymbol{v}$ being zero for a gas at rest). The particle number density of species $k$ is denoted by $n_{k}, H_{k}^{\text {rot }}$ is the internal rotational Hamiltonian and $Z_{k}^{\text {rot }}$ is the corresponding rotational partition function.

The linearized Waldmann-Snider equation for the relative deviation $\Phi_{k}$ of the distribution of species $k$ from equilibrium is

$$
\frac{\partial \Phi_{k}}{\partial t}+\boldsymbol{c}_{k} \cdot \boldsymbol{\nabla} \Phi_{k}+\sum_{l} \omega_{k l}\left(\Phi_{l}\right)=0 .
$$

The linearized collision operator $\omega_{k l}$ can be split into two parts

$$
\omega_{k l}=\delta_{k l} \sum_{m} \omega_{k m}^{(1)}+\omega_{k l}^{(2)},
$$

where, in a short-hand notation, $\omega_{k m}^{(1)}$ and $\omega_{k l}^{(2)}$ are given by

$$
\begin{aligned}
& \omega_{k m}^{(1)}\left(\Phi_{l}\right)=-\operatorname{Tr}_{2} \int \mathrm{d}^{3} c_{2} f_{m}^{(0)}(2) \\
& \quad \cdot\left\{\int a_{k m} \Phi_{k}^{\prime}(1) a_{k m}^{\dagger} g^{\prime} \mathrm{d}^{2} e^{\prime}\right. \\
& \left.\quad-\frac{h}{i m_{k m}}\left[a_{k m}(0) \Phi_{k}(1)-\Phi_{k}(1) a_{k m}^{\dagger}(0)\right]\right\} \delta_{k l},
\end{aligned}
$$

and

$$
\begin{aligned}
\omega_{k l}^{(2)}\left(\Phi_{l}\right)= & -\operatorname{Tr}_{2} \int \mathrm{d}^{3} c_{2} f_{l}^{(0)}(2) \\
& \cdot\left\{\int a_{k l} \Phi_{l}{ }^{\prime}(2) a_{k l}^{\dagger} g^{\prime} \mathrm{d}^{2} e^{\prime}\right. \\
& \left.-\frac{h}{i m_{k l}}\left[a_{k l}(0) \Phi_{l}(2)-\Phi_{l}(2) a_{k l}^{\dagger}(0)\right]\right\} .
\end{aligned}
$$

Here, $\operatorname{Tr}_{2}$ denotes the trace over the internal rotational states of the second particle, the prime (e.g. $\left.\Phi_{k}{ }^{\prime}(1)\right)$ means that precollisional variables have to be taken, and $g^{\prime} \boldsymbol{e}^{\prime}$ is the relative velocity ( $g^{\prime}$ magnitude, $\boldsymbol{e}^{\prime}$ direction) before the collision. The quantity $a_{k m}\left(\boldsymbol{e}, \boldsymbol{e}^{\prime}\right)$ is the binary scattering operator on the energy shell describing collisions between molecules of species $k$ and $m, a_{k m}^{\dagger}$ is its adjoint in internal state space and $a_{k m}(0)$ is the scattering amplitude in the forward direction $\boldsymbol{e}=\boldsymbol{e}^{\prime} ; m_{k l}$ is the reduced mass. Note that in a representation $\Phi_{k}$ is diagonal with respect to rotational quantum numbers but not $a_{k m}$ (inelastic collisions!). The scattering amplitude is linked with the $T$-operator by

$$
a_{k l}\left(\boldsymbol{k}, \boldsymbol{k}^{\prime}\right)=-\frac{m_{k l}}{2 \pi \hbar^{2}}\left\langle\boldsymbol{k}\left|T_{k l}\right| \boldsymbol{k}^{\prime}\right\rangle,
$$

where $\boldsymbol{k}^{\prime}=k^{\prime} \boldsymbol{e}^{\prime}, \quad \boldsymbol{k}=k \boldsymbol{e}$ are the relative wave vectors before and after the collision, respectively. The binary scattering operator $T_{k l}$ is connected with the nonspherical intermolecular potential $V_{k l}$ by the scattering integral equation [24]

$$
T_{k l}=V_{k l}+V_{k l}\left(E-H_{k l}^{(0)}+i \eta\right)^{-1} T_{k l},
$$

where $H_{k l}^{(0)}$ is the free particle Hamiltonian in the c.m. system and $\eta \rightarrow 0^{+}$. The optical theorem for $T_{k l}$,

$$
\begin{aligned}
T_{k l}-T_{k l}^{\dagger} & =-2 \pi i T_{k l} \delta\left(E-H_{k l}^{(0)}\right) T_{k l}^{\dagger} \\
& =-2 \pi i T_{k l}^{\dagger} \delta\left(E-H_{k l}^{(0)}\right) T_{k l}(
\end{aligned}
$$


leads to the two formulations of the optical theorem for $a_{k l}$ :

$$
\begin{aligned}
\frac{h}{i m_{k l}} & {\left[a_{k l}\left(\boldsymbol{k}=\boldsymbol{k}^{\prime}\right)-a_{k l}^{\dagger}\left(\boldsymbol{k}=\boldsymbol{k}^{\prime}\right)\right] } \\
& =\int a_{k l}\left(\boldsymbol{k}, \boldsymbol{k}^{\prime}\right) a_{k l}^{\dagger}\left(\boldsymbol{k}, \boldsymbol{k}^{\prime}\right) g^{\prime} \mathrm{d}^{2} e^{\prime} \\
& =\int a_{k l}^{\dagger}\left(\boldsymbol{k}^{\prime}, \boldsymbol{k}\right) a_{k l}\left(\boldsymbol{k}^{\prime}, \boldsymbol{k}\right) g^{\prime} \mathrm{d}^{2} e^{\prime} .
\end{aligned}
$$

Equations (1.10) are of great importance for the formulation of conservation laws, positive semidefiniteness and explicit expressions for the collision integrals.

Collision brackets of two arbitrary quantities $\Psi$ and $\Phi$ are defined by

$\left\langle\left\langle\Psi \omega^{(1)}(\Phi)\right\rangle\right\rangle_{k l}=\operatorname{Tr} \int \mathrm{d}^{3} c \Psi_{k}^{\dagger} \frac{f_{k}^{(0)}}{n_{k}} \omega_{k l}^{(1)}\left(\Phi_{l}\right)$,

and correspondingly for $\left\langle\left\langle\Psi \omega^{(2)}(\Phi)\right\rangle\right\rangle_{k l}$. According to Eq. (1.4), the total collision bracket is given by

$$
\begin{aligned}
& \langle\langle\Psi \omega(\Phi)\rangle\rangle_{k l} \\
& \quad=\delta_{k l} \sum_{m}\left\langle\left\langle\Psi \omega^{(1)}(\Phi)\right\rangle\right\rangle_{k m}+\left\langle\left\langle\Psi \omega^{(2)}(\Phi)\right\rangle\right\rangle_{k l} .
\end{aligned}
$$

For Hermitian $\Psi, \Phi$, the collision brackets are real.

\section{Semi-Positiveness, Collision Brackets of Summational Invariants}

Most of the properties listed herein have already been discussed in Ref. [8], thus a brief review is sufficient here. Using the optical theorem Eqs. (1.10), we can show after some manipulations that one has

$$
\begin{gathered}
\left\langle\left\langle\Phi \omega^{(1)}(\Phi)\right\rangle\right\rangle_{k l}=\frac{1}{4}\left(n_{k}\right)^{-1} \operatorname{Tr}_{1} \operatorname{Tr}_{2} \\
\cdot \iiint \mathrm{d}^{3} c_{1} \mathrm{~d}^{3} c_{2} g^{\prime} \mathrm{d}^{2} e^{\prime} f_{k}^{(0)}(1) f_{k}^{(0)}(2) \\
\left|\Phi_{k}(1) a_{k l}-a_{k l} \Phi_{k}^{\prime}(1)\right|^{2},
\end{gathered}
$$

which is obviously non-negative. For $\Phi_{k}=1$, the r.h.s. of Eqs. (1.13) gives zero, which, after Eq. (1.3), yields the equation of continuity. For the following considerations it is convenient to use the operator [13]

$$
R_{k l} \equiv\left(n_{k} / n^{2}\right) \omega_{k l},
$$

where $n$ is the total particle number density. Similar manipulations [8] which lead to Eq. (1.13) also yield the inequality

$$
\sum_{k, l}\langle\langle\Phi R(\Phi)\rangle\rangle_{k l} \geqq 0,
$$

which guarantees the increase of entropy in the linearized theory. For $\Phi$ 's not depending on the vector $J$, one obtains, using Eqs. (1.4), (1.5), (1.10), (1.11), (1.12), for arbitrary $\Psi$, the relation

$$
\begin{aligned}
& \sum_{l}\langle\langle\Psi R(\Phi)\rangle\rangle_{k l}=n^{-2} \operatorname{Tr}_{1} \operatorname{Tr}_{2} \iint \mathrm{d}^{3} c_{1} \mathrm{~d}^{3} c_{2} \\
& \cdot \sum_{l} f_{k}(1) f_{l}(2) \int g^{\prime} \mathrm{d}^{2} e^{\prime} \Psi_{k}(1) \\
& \cdot a_{k l}\left(\Phi_{k}(1)+\Phi_{l}(2)-\Phi_{k}{ }^{\prime}(1)-\Phi_{l}{ }^{\prime}(2)\right) a_{k l}^{\dagger} .
\end{aligned}
$$

From Eq. (1.15) one infers that for summational invariants $\Phi_{\text {inv }}$, i.e. quantities fulfilling

$$
\Phi_{k}(1)+\Phi_{l}(2)-\Phi_{k}{ }^{\prime}(1)-\Phi_{l^{\prime}}(2)=0
$$

the relation

$$
\sum_{l}\left\langle\left\langle\Psi R\left(\Phi_{\mathrm{inv}}\right)\right\rangle\right\rangle_{k l}=0
$$

holds. Summational invariants of this kind are 1, $m \boldsymbol{c}$ and $m c^{2} / 2+E^{\text {int }}$. In the same way also the relation

$$
\sum_{k}\left\langle\left\langle\Phi_{\mathrm{inv}} R(\Psi)\right\rangle\right\rangle_{k l}=0
$$

can be derived which is of importance for the formulation of the linearized conservation laws.

\section{Properties Following from Rotational-, Parity-and Time-Reversal Invariance of Molecular Interaction}

The intermolecular potential is rotationally-and parity invariant. If the influence of external fields on the collision process can be neglected, the collision operator is a scalar. If chiral molecules are not considered here, the collision operator also conserves parity. Thus one has for two spherical tensor operators $\left[\Phi^{\mathrm{P}}(\boldsymbol{W}, \boldsymbol{J})\right]_{M}^{L}$ and $\left[\Psi^{\mathrm{P}}(\boldsymbol{W}, \boldsymbol{J})\right]_{\boldsymbol{M}^{\prime}}^{L^{\prime}}(\mathrm{P}=$ parity $)$ according to the Wigner-Eckart theorem:

$$
\left\langle\left\langle\left[\Phi \Phi^{\mathrm{P}}\right]_{M}^{L} R\left(\left[\Psi^{\mathrm{P}^{\prime}}\right]_{M^{\prime}}^{L^{\prime}}\right)\right\rangle\right\rangle_{k l}=\frac{1}{2 L+1}\left\langle\left\langle\left[\Phi^{\mathrm{P}}\right]^{\mathrm{L}} \circ R\left(\left[\Psi^{\mathrm{P}}\right]^{\mathrm{L}}\right)\right\rangle\right\rangle_{k l} \delta^{\mathrm{PP}^{\prime}} \delta_{L L^{\prime}} \delta_{M M^{\prime}},
$$

where the dot denotes a contraction over all tensor indices.

Finally the time reversal invariance of molecular interaction (expressed by $\vec{a}_{k l}\left(\boldsymbol{k}, \boldsymbol{k}^{\prime}\right)=\overleftarrow{a}_{k l}\left(-\boldsymbol{k}^{\prime},-\boldsymbol{k}\right)$ where the arrows show the direction in which $a_{k l}$ is acting) immediately leads to the Onsager relations

$$
\langle\langle\Phi R(\Psi)\rangle\rangle_{k l}=\left\langle\left\langle\Psi_{T} R\left(\Phi_{T}\right)\right\rangle\right\rangle_{l k},
$$


where $\Phi_{T}(\boldsymbol{W}, \boldsymbol{J}) \equiv \Phi(-\boldsymbol{W},-\boldsymbol{J})$. If $\Phi_{T}=T_{\Phi} \Phi$ and $\Psi_{T}=T_{\Psi} \Psi$ with $T_{\Phi, \Psi}= \pm 1$, one has

$$
\langle\langle\Phi R(\Psi)\rangle\rangle_{k l}=T_{\Phi} T_{\Psi}\langle\langle\Psi R(\Phi)\rangle\rangle_{l k} .
$$

Since the product $T_{\Phi} T_{\Psi}$ cal also be -1 , the linearized Waldmann-Snider collision operator is - different from the Boltzmann collision operator - in general not self-adjoint.

\section{Effective Cross Sections. Exact Relations}

\section{Definition of Effective Cross Sections}

We are now considering matrix elements of the collision superoperator $R_{k l}$ for binary mixtures constructed with a basis set of compound expansion tensors $\Phi^{(p q r s \mid k)}(\boldsymbol{W}, \boldsymbol{J})$. Here, $p$ is the tensor rank in the peculiar velocity $\boldsymbol{W}, q$ is the tensor rank in $\boldsymbol{J} ; r$ and $s$ are the ranks of the Sonine polynomials in $W^{2}$ and Wang-Chang-Uhlenbeck polynomials in $\left(J^{2}-\left\langle J^{2}\right\rangle_{0}\right)$. While this notation is complete for gases of linear molecules, for symmetric top molecules a further superscript $t$ is necessary which stands for the power in $J_{\|}$, the component of $\boldsymbol{J}$ along the figure axis. In a spherical tensor representation $\left(\Phi_{m n}^{(p q r s \mid k)}\right.$, $-p \leqq m \leqq p,-q \leqq n \leqq q$, Edmonds' [25] convention being used) a general matrix element of the collision operator is, in the coupling scheme of Chen, Moraal and Snider [17], given by

$$
\begin{aligned}
& \left\langle\left\langle\Phi_{m n}^{(p q r s \mid k)} R\left(\Phi_{m^{\prime} n^{\prime}}^{\left(p^{\prime} q^{\prime} r^{\prime} s^{\prime} \mid l\right)}\right)\right\rangle\right\rangle_{k l}=i^{p-p^{\prime}+q-q^{\prime}} \sum_{K, \varkappa}(-1)^{K+p+q+m+n^{\prime}} \Omega\left(K q^{\prime} q\right)^{1 / 2} \Omega\left(K p^{\prime} p\right)^{1 / 2} \\
& \cdot\left(\begin{array}{ccc}
K & q & q^{\prime} \\
\varkappa & -n & n^{\prime}
\end{array}\right)\left(\begin{array}{ccc}
K & p & p^{\prime} \\
-\varkappa & -m & m^{\prime}
\end{array}\right) S_{K}\left(\begin{array}{ccc|c}
p & q & r & s \\
p^{\prime} q^{\prime} r^{\prime} s^{\prime} & l
\end{array}\right),
\end{aligned}
$$

where $\left(\begin{array}{ccc}K & q & q^{\prime} \\ \varkappa & -n & n^{\prime}\end{array}\right)$ is a $3 j$-symbol [25] and $\Omega$ is given by

$\Omega\left(K p^{\prime} p\right)=\frac{\left(p+p^{\prime}+K+1\right) !\left(p+p^{\prime}-K\right) !\left(p-p^{\prime}+K\right) !\left(p^{\prime}-p+K\right) !}{(2 K) !(2 p) !\left(2 p^{\prime}\right) !} \frac{3-(-1)^{p+p^{\prime}+K}}{2}$

The scalar quantities (,,reduced matrix elements") $S_{K}$ yield the information about molecular dynamics.

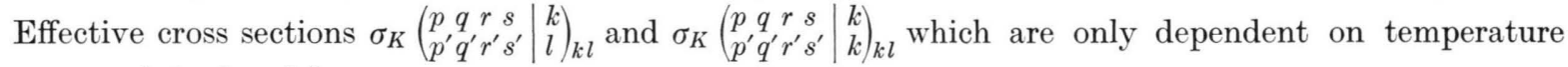
are now introduced by

$$
\begin{aligned}
& S_{K}\left(\begin{array}{ccc|c}
p & q & r & s \\
p^{\prime} q^{\prime} r^{\prime} s^{\prime} & k \\
l
\end{array}\right)=x_{k} x_{l} v_{k l} \sigma_{K}\left(\begin{array}{ccc|c}
p & q & r & s \\
p^{\prime} q^{\prime} r^{\prime} s^{\prime} & k \\
l
\end{array}\right)_{k l}, \quad k \neq l,
\end{aligned}
$$

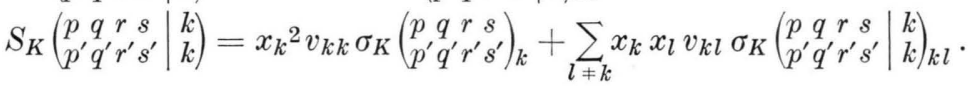

In Eq. (2.3), $x_{k}=n_{k} / n$ is the mole fraction of species $k, v_{k l}=\left(8 k_{\mathrm{B}} T / \pi m_{k l}\right)^{1 / 2}$ is a thermal velocity and

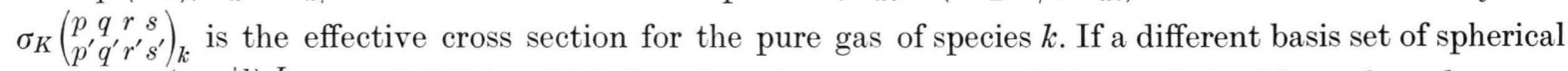
tensors $\left[\Phi^{(p q r s \mid k)}\right]_{M}^{L}$ is used [26] in which first the $p$ th rank tensor in $\boldsymbol{W}$ is coupled with a $q$ th rank tensor in $\boldsymbol{J}$ to give a totally irreducible $L$ th rank spherical tensor (coupling scheme of Hess, Waldmann, Köhler,

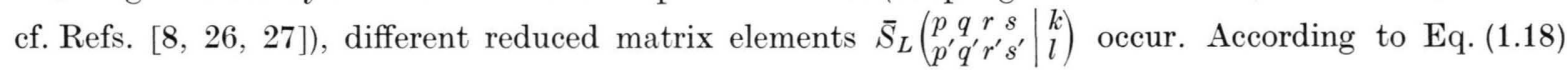
one has

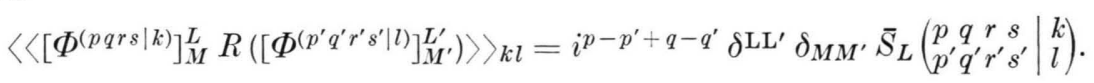

The corresponding cross sections $\bar{\sigma}_{L}(\cdots)$ are defined analogously to Equation (2.3).

Both kinds of quantities $S_{K}$ and $\bar{S}_{L}$ are connected with each other by [28]

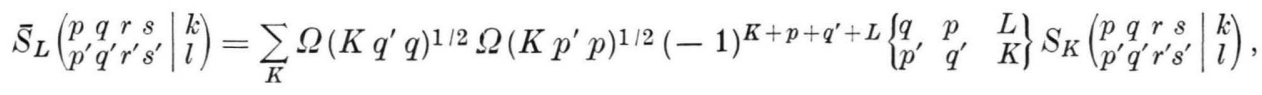


and, vice versa,

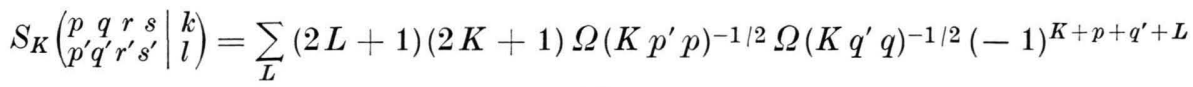

$$
\begin{aligned}
& \cdot\left\{\begin{array}{lll}
q & p & L \\
p^{\prime} & q^{\prime} & K
\end{array}\right\} \bar{S}_{L}\left(\begin{array}{ccc|c}
p & q & r & s \\
p^{\prime} q^{\prime} r^{\prime} s^{\prime} & l & l
\end{array}\right),
\end{aligned}
$$

$\left\{\begin{array}{lll}q & p & L \\ p^{\prime} & q^{\prime} & K\end{array}\right\}$ being a $6 j$-symbol $[25]$.

\section{Bracket Symbols}

The effective Waldmann-Snider cross sections form the link between experiment and theory. They can on the one hand be extracted from experimental data (e.g. magnitudes of Senftleben-Beenakker effects, line widths etc.) on the other hand evaluated theoretically if the binary scattering amplitude is known. To get more insight into the structure of these effective cross sections (which are much more complex than the corresponding ones for noble gases) it is necessary to reduce the lengthy expressions as far as possible and to introduce a lucid notation. First it is convenient to introduce dimensionless variables: a dimensionless c.m. velocity $\boldsymbol{V}$ and dimensionless relative velocities before and after the collision $\boldsymbol{\gamma}^{\prime}=\boldsymbol{\gamma}^{\prime} \boldsymbol{e}^{\prime}$ and $\boldsymbol{\gamma}=\boldsymbol{\gamma} \boldsymbol{e}$, respectively. They are connected with the velocities of the colliding molecules before the collision by

$$
\begin{aligned}
& \boldsymbol{c}_{1}{ }^{\prime}=\left(\frac{2 k_{\mathrm{B}} T}{m_{k}}\right)^{1 / 2}\left[\left(\frac{m_{k}}{m_{k}+m_{l}}\right)^{1 / 2} \boldsymbol{V}+\left(\frac{m_{l}}{m_{k}+m_{l}}\right)^{1 / 2} \gamma^{\prime} \boldsymbol{e}^{\prime}\right], \\
& \boldsymbol{c}_{2}{ }^{\prime}=\left(\frac{2 k_{\mathrm{B}} T}{m_{l}}\right)^{1 / 2}\left[\left(\frac{m_{l}}{m_{k}+m_{l}}\right)^{1 / 2} \boldsymbol{V}-\left(\frac{m_{k}}{m_{k}+m_{l}}\right)^{1 / 2} \gamma^{\prime} \boldsymbol{e}^{\prime}\right] .
\end{aligned}
$$

For the postcollisional (unprimed) variables $\gamma^{\prime} \boldsymbol{e}^{\prime}$ has to be replaced by $\gamma \boldsymbol{e}$. Energy conservation in a collision requires that

$$
\gamma^{2}+\Delta \varepsilon_{k l}=\gamma^{\prime 2}
$$

holds where $\Delta \varepsilon_{k l}$ is the transfer in internal energy $/ k_{\mathrm{B}} T$; for linear molecules, which are mainly considered here, one has

$$
\Delta \varepsilon_{k l}=\varepsilon_{k}\left(j_{1}\right)+\varepsilon_{l}\left(j_{2}\right)-\varepsilon_{k}\left(j_{1}{ }^{\prime}\right)-\varepsilon_{l}\left(j_{2}{ }^{\prime}\right),
$$

where $\varepsilon_{k}(j)=\hbar^{2} j(j+1) / 2 \Theta_{k} k_{\mathrm{B}} T$ and $j_{1}{ }^{\prime} j_{2}{ }^{\prime}\left(j_{1} j_{2}\right)$ are the precollisional (postcollisional) rotational quantum numbers. Using these dimensionless variables, we find e.g. for $\bar{\sigma}_{L}\left(\begin{array}{lll|l}p & q & r & s \\ p^{\prime} & q^{\prime} & r^{\prime} & s^{\prime}\end{array} \mid \begin{array}{l}k \\ k\end{array}\right)_{k l}$ in a detailed notation:

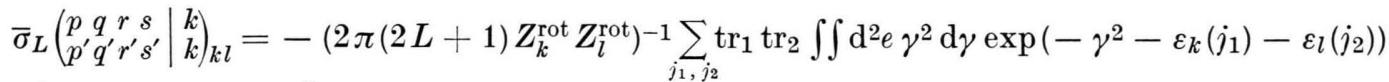

$$
\begin{aligned}
& \cdot\left\{\Phi _ { L } ^ { j _ { 1 } ( p q r s | k ) } ( \boldsymbol { V } , \boldsymbol { \gamma } \boldsymbol { e } ) \left[\sum_{j_{1} j_{2}{ }^{\prime}} \int a_{k l}^{j_{1} j_{2}, j_{1}{ }^{\prime} j_{2}{ }^{\prime}}\left(\gamma \boldsymbol{e}, \gamma^{\prime} \boldsymbol{e}^{\prime}\right) \Phi_{L}^{j_{1}{ }^{\prime}\left(p^{\prime} q^{\prime} r^{\prime} s^{\prime} \mid k\right)}\left(\boldsymbol{V}, \boldsymbol{\gamma}^{\prime} \boldsymbol{e}^{\prime}\right) a_{k l}^{\dagger j_{1}{ }^{\prime} j_{2}{ }^{\prime}, j_{1} j_{2}}\left(\gamma \boldsymbol{e}, \gamma^{\prime} \boldsymbol{e}^{\prime}\right) \gamma^{\prime} \mathrm{d}^{2} e^{\prime}\right.\right. \\
& \left.\left.-\frac{h}{i \sqrt{2 m_{k l} k_{\mathrm{B}} T}}\left(a_{k l}^{j_{1} j_{2}, j_{1} j_{2}}(\gamma \boldsymbol{e}, \gamma \boldsymbol{e}) \Phi_{L}^{j_{1}\left(p^{\prime} q^{\prime} r^{\prime} s^{\prime} \mid k\right)}(\boldsymbol{V}, \gamma e)-\Phi_{L}^{j_{1}\left(p^{\prime} q^{\prime} r^{\prime} s^{\prime} \mid k\right)}(\boldsymbol{V}, \boldsymbol{\gamma} \boldsymbol{e}) a_{k l}^{\dagger j_{1} j_{2}, j_{1} j_{2}}(\gamma \boldsymbol{e}, \gamma \boldsymbol{e})\right)\right]\right\} .
\end{aligned}
$$

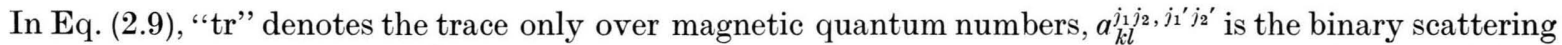
amplitude (which is, like $\Phi_{L}^{j_{1}}$, a matrix with respect to magnetic quantum numbers) for the collision process $j_{1}{ }^{\prime}+j_{2}{ }^{\prime} \rightarrow j_{1}+j_{2}$. The curly bracket symbol $\{\cdots\}$ means an average with respect to the c.m. velocity:

$$
\{\cdots\} \equiv \pi^{-3 / 2} \int \exp \left(-V^{2}\right)(\cdots) \mathrm{d}^{3} V .
$$

Since the scattering amplitude does not depend on $\boldsymbol{V}$, this average can always be performed explicitly. After evaluation of the traces, the integrand as a scalar can only depend on the scalars $\gamma, \gamma^{\prime}$, and $\cos \vartheta$ 
$=\boldsymbol{e} \cdot \boldsymbol{e}^{\prime}$ (scattering angle $\vartheta$ ). Therefore the double integral $\iint \mathrm{d}^{2} e \mathrm{~d}^{2} e^{\prime} \ldots$ can be replaced by a single angular integral $8 \pi^{2} \int \sin \vartheta \mathrm{d} \vartheta \ldots$. Thus it is useful to introduce a second bracket symbol

$$
\llbracket \cdots \rrbracket \equiv 2 \pi\left(Z_{k}^{\text {rot }} Z_{l}^{\text {rot }}\right)^{-1} \sum_{j_{1} j_{2} j_{1} j_{2}{ }^{\prime}}\left(2 j_{1}+1\right)\left(2 j_{2}+1\right) \iint \sin \vartheta \mathrm{d} \vartheta \gamma^{3} \mathrm{~d} \gamma(\cdots) \exp \left(-\varepsilon_{k}\left(j_{1}\right)-\varepsilon_{l}\left(j_{2}\right)-\gamma^{2}\right)
$$

which involves an average over $\gamma$ and postcollisional rotational quantum numbers as well as an integration over the scattering angle and a sum over precollisional rotational quantum numbers.

\section{Expressions for Specific Effective Cross Sections. Exact Relations}

For specific expansion tensors $\Phi^{(p q r s \mid k)}, \Phi^{\left(p^{\prime} q^{\prime} r^{\prime} s^{\prime} \mid l\right)}$, the average Eq. (2.10) can always be performed explicitly, the corresponding effective cross section is then represented in terms of a bracket symbol (2.11). After this reduction already interesting non-trivial relations between effective cross sections can be found. They can be used to reduce the number of unknown cross sections to be determined from experiments with mixtures of polyatomics.

The system of specific expansion tensors under consideration is shown in Table 1. The system chosen has proved to be sufficient for a complete kinetic description of all transport and relaxation phenomena in mixtures of dilute polyatomic gases studied up to now. We call those tensors which solely depend on $\boldsymbol{W}_{k}$ and $J_{k}^{2}$ ( $\propto \varepsilon_{k}$ for linear molecules) "classical" ones since they commute with $\boldsymbol{J}_{k}$ and with the scattering amplitude if projected on $j$ subspace. The remaining ones which don't commute with $\boldsymbol{J}_{k}$ are called "quantum mechanical" ones.

Let us first consider effective cross sections describing the relaxation and coupling of "classical" expansion tensors. From Eqs. (2.9), the optical theorem (1.10) and Eq. (2.11) one obtains generally:

$$
\sigma\left(\begin{array}{ccc|c}
p & 0 & r & s \\
p^{\prime} & 0 & r^{\prime} s^{\prime} & k \\
k
\end{array}\right)_{k l}=\frac{2 \delta_{p p^{\prime}}}{2 p+1} \llbracket \sigma_{k l}\left\{\Phi^{(p 0 r s \mid k)} \circ\left(\Phi^{\left(p^{\prime} 0 r^{\prime} s^{\prime} \mid k\right)}-\Phi^{\prime\left(p^{\prime} 0 r^{\prime} s^{\prime} \mid k\right)}\right)\right\} \rrbracket,
$$

and

$$
\sigma\left(\begin{array}{ccc|c}
p & 0 & r & s \\
p^{\prime} & 0 & r^{\prime} s^{\prime} & k \\
l
\end{array}\right)_{k l}=\frac{2 \delta_{p p^{\prime}}}{2 p+1} \llbracket \sigma_{k l}\left\{\Phi^{(p 0 r s \mid k)} \circ\left(\Phi^{\left(p^{\prime} 0 r^{\prime} s^{\prime} \mid l\right)}-\Phi^{\prime\left(p^{\prime} 0 r^{\prime} s^{\prime} \mid l\right)}\right)\right\} \rrbracket .
$$

Herein, the molecular cross section

$$
\sigma_{k l} \equiv\left[\left(2 j_{1}+1\right)\left(2 j_{2}+1\right)\right]^{-1} \frac{\gamma^{\prime}}{\gamma} \operatorname{tr}_{1} \operatorname{tr}_{2}\left(a_{k l}^{j_{1} j_{2}, j_{1}{ }^{\prime} j_{2}{ }^{\prime}} a_{k l}^{+j_{1}{ }^{\prime} j_{2}{ }^{\prime}, j_{1} j_{2}}\right)
$$

is the unpolarized differential cross section for the (inverse) collision process $j_{1}, j_{2}, \gamma \rightarrow j_{1}{ }^{\prime}, j_{2}{ }^{\prime}, \gamma^{\prime}$ averaged over initial and summed over final magnetic quantum numbers.

Table 1. Set of orthonormal expansion tensors for polyatomic gases.

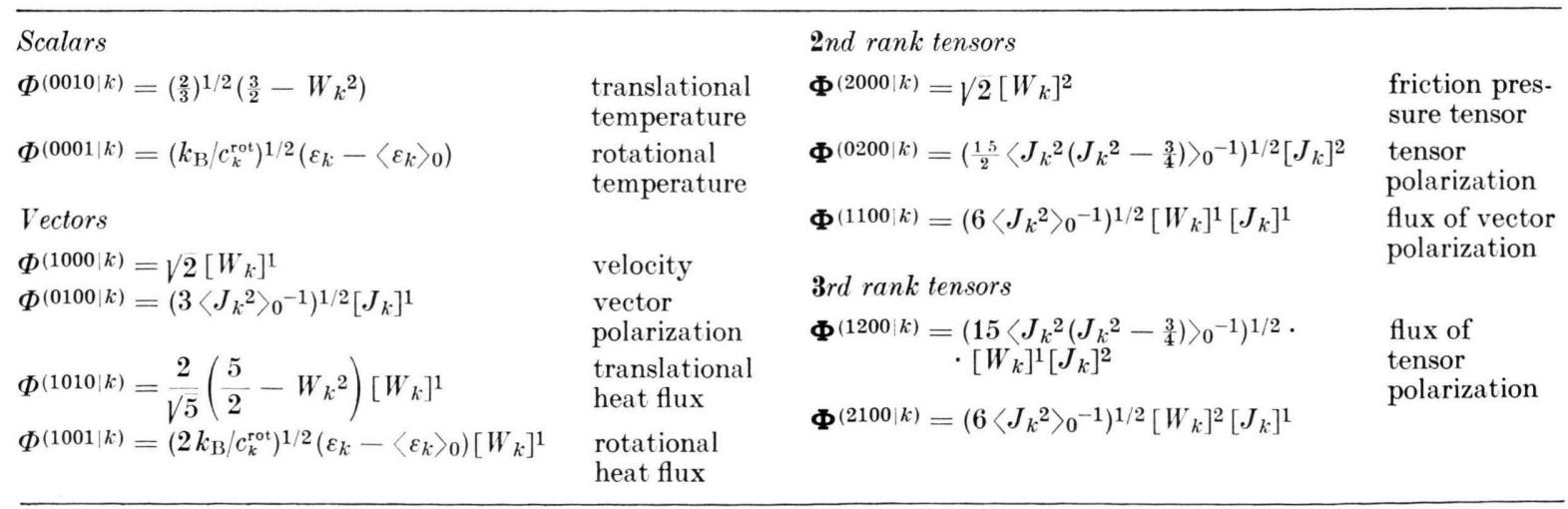


Evaluation of the c.m. average in Eqs. (2.12), (2.13) yields for the effective cross sections of the scalars in Table 1 the expressions:

$$
\begin{aligned}
& \sigma\left(\begin{array}{l|l}
0010 & k \\
0010 & k
\end{array}\right)_{k l}=\frac{2}{3}\left(\frac{m_{l}}{m_{k}+m_{l}}\right)^{2} \llbracket \sigma_{k l}\left(\left(\Delta \varepsilon_{k l}\right)^{2}+\frac{2 m_{k}}{m_{l}}\left(\gamma^{2}+\gamma^{\prime 2}-2 \gamma \gamma^{\prime} \cos \vartheta\right)\right) \rrbracket, \\
& \sigma\left(\begin{array}{c|l}
0010 & k \\
0010 & l
\end{array}\right)_{k l}=\frac{2}{3} \frac{m_{k} m_{l}}{\left(m_{k}+m_{l}\right)^{2}} \llbracket \sigma_{k l}\left(\left(\Delta \varepsilon_{k l}\right)^{2}-2\left(\gamma^{2}+\gamma^{\prime 2}-2 \gamma \gamma^{\prime} \cos \vartheta\right)\right) \rrbracket, \\
& \sigma\left(\begin{array}{c|c}
0001 & k \\
0001 & k
\end{array}\right)_{k l}=\frac{k_{\mathrm{B}}}{c_{k}^{\text {rot }} \llbracket \sigma_{k l}\left(\varepsilon_{k}-\varepsilon_{k}\right)^{\prime} \rrbracket,} \\
& \sigma\left(\begin{array}{c|c}
0001 & k \\
0001 & l
\end{array}\right)_{k l}=\frac{k_{\mathrm{B}}}{\sqrt{c_{k}^{\text {rot }} c_{l}^{\text {rot }}} \llbracket \sigma_{k l}\left(\varepsilon_{k}-\varepsilon_{k}{ }^{\prime}\right)\left(\varepsilon_{l}-\varepsilon_{l}^{\prime}\right) \rrbracket .}
\end{aligned}
$$

These cross sections determine the relaxation of translational and rotational temperatures. The last two ones are purely inelastic since only cross sections which are connected with a change in internal energy can contribute. Conservation of total energy in a collision yields (after Eqs. $(1.16,1.17)$ for arbitrary $p q r s)$ :

$$
-\sqrt{\frac{3}{2}} \sigma\left(\begin{array}{c|c}
0010 \\
p q r s
\end{array} \mid \begin{array}{l}
k \\
k
\end{array}\right)_{k l}+\sqrt{\frac{c_{k}^{\text {rot }}}{k_{\mathrm{B}}}} \sigma\left(\begin{array}{c|c|c}
0001 \\
p q r s
\end{array} \mid \begin{array}{l}
k \\
k
\end{array}\right)_{k l}-\sqrt{\frac{3}{2}} \sigma\left(\begin{array}{c}
0010 \\
p q r s
\end{array} \mid \begin{array}{l}
l \\
k
\end{array}\right)_{k l}+\sqrt{\frac{c_{l}^{\mathrm{rot}}}{k_{\mathrm{B}}}} \sigma\left(\begin{array}{l}
0001 \\
p q r s
\end{array} \mid \begin{array}{l}
l \\
k
\end{array}\right)_{k l}=0 .
$$

A corresponding relation where the upper and lower row of arguments in the $\sigma$ 's of Eq. (2.19) are interchanged is also valid.

The relaxation cross section for the velocity which determines the isotropic diffusion coefficient [13] is given by

$$
\sigma\left(\begin{array}{l|l}
1000 & k \\
1000 & k
\end{array}\right)_{k l}=\frac{2}{3} \frac{m_{l}}{m_{k}+m_{l}} \llbracket \sigma_{k l}\left(\gamma^{2}+\gamma^{\prime 2}-2 \gamma \gamma^{\prime} \cos \vartheta\right) \rrbracket .
$$

From conservation of linear momentum an exact relation follows for arbitrary pqrs:

$$
\sigma\left(\begin{array}{l|l}
1000 & l \\
p q r s & k
\end{array}\right)_{k l}=-\sqrt{\frac{m_{k}}{m_{l}}} \sigma\left(\begin{array}{c|c}
1000 & k \\
p q r s & k
\end{array}\right)_{k l} .
$$

Again, upper and lower rows in the $\sigma$ 's may be interchanged. The coupling cross sections between velocity and the heat flux vectors which are essential for the thermal diffusion coefficient [13] are found as

$$
\begin{aligned}
& \sigma\left(\begin{array}{l|l}
1000 & k \\
1010 & k \\
k
\end{array}\right)_{k l}=-\frac{4}{3} \sqrt{\frac{2}{5}}\left(\frac{m_{l}}{m_{k}+m_{l}}\right)^{2} \llbracket \sigma_{k l}\left(\gamma^{2}-{ }_{2}^{5}\right)\left(\gamma^{2}-\gamma \gamma^{\prime} \cos \vartheta\right) \rrbracket, \\
& \sigma\left(\begin{array}{l|l}
1000 & k \\
1010 & l
\end{array}\right)_{k l}=-\left(m_{k} / m_{l}\right)^{3 / 2} \sigma\left(\begin{array}{c}
1000 \\
1010
\end{array} \mid \begin{array}{l}
k \\
k
\end{array}\right)_{k l}, \\
& \sigma\left(\begin{array}{c|c}
1000 \\
1001
\end{array} \mid \begin{array}{l}
k \\
k
\end{array}\right)_{k l}=\frac{4}{3} \sqrt{\frac{k_{\mathrm{B}}}{c_{k}^{\text {rot }}}} \frac{m_{l}}{m_{k}+m_{l}} \llbracket \sigma_{k l}\left(\varepsilon_{k}-\left\langle\varepsilon_{k}\right\rangle_{0}\right)\left(\gamma^{2}-\gamma \gamma^{\prime} \cos \vartheta\right) \rrbracket .
\end{aligned}
$$

The cross section (2.23) vanishes for a purely spherical molecular interaction because in this case $\sigma_{k l}$ is $j$-independent and the $j$-average of $\varepsilon_{k}-\left\langle\varepsilon_{k}\right\rangle_{0}$ yields zero.

The effective cross sections of the heat flux vectors determining isotropic heat conduction [13] are somewhat lengthy expressions. For the translational heat flux one finds

$$
\begin{aligned}
\sigma\left(\begin{array}{l|l}
1010 \\
1010
\end{array} \mid \begin{array}{l}
k \\
k
\end{array}\right)_{k l}= & \frac{1}{3}\left(\frac{m_{l}}{m_{k}+m_{l}}\right)^{2} \llbracket \sigma_{k l}\left(\left(\gamma^{2}+\gamma^{\prime 2}-2 \gamma \gamma^{\prime} \cos \vartheta\right)\left(11\left(m_{k l} m_{k} / m_{l}{ }^{2}\right)-5\left(m_{k} / m_{l}\right)+5\right)\right. \\
& -4\left(m_{k l} / m_{k}\right)\left(\gamma^{4}+\gamma^{4}-\left(\gamma^{2}+\gamma^{\prime 2}\right) \gamma \gamma^{\prime} \cos \vartheta\right)+\frac{4}{5}\left(m_{k l} / m_{k}\right)\left(\gamma^{6}+\gamma^{\prime 6}-2 \gamma^{3} \gamma^{\prime 3} \cos \vartheta\right) \\
& \left.+\frac{14}{5}\left(m_{k l} / m_{l}\right)\left(\Delta \varepsilon_{k l}\right)^{2}+\frac{8}{5}\left(m_{k l} / m_{l}\right)\left(\gamma^{4}+\gamma^{\prime 4}-2 \gamma^{2} \gamma^{\prime 2} \cos ^{2} \vartheta\right)\right) \rrbracket,
\end{aligned}
$$




$$
\begin{aligned}
\sigma\left(\begin{array}{l|l}
1010 \\
1010
\end{array} \mid \begin{array}{l}
k \\
l
\end{array}\right)_{k l}= & \frac{1}{3}\left(\frac{m_{k} m_{l}}{\left(m_{k}+m_{l}\right)^{2}}\right)^{3 / 2} \llbracket \sigma_{k l}\left(-11\left(\gamma^{2}+\gamma^{\prime 2}-2 \gamma \gamma^{\prime} \cos \vartheta\right)+4\left(\gamma^{4}+\gamma^{\prime 4}\right.\right. \\
& \left.-\left(\gamma^{2}+\gamma^{\prime 2}\right) \gamma \gamma^{\prime} \cos \vartheta\right)-\frac{4}{5}\left(\gamma^{6}+\gamma^{\prime 6}+2 \gamma^{3} \gamma^{\prime 3} \cos \vartheta\right)+\frac{14}{5}\left(\Delta \varepsilon_{k l}\right)^{2} \\
& \left.+\frac{8}{5}\left(\gamma^{4}+\gamma^{\prime}-2 \gamma^{2} \gamma^{\prime 2} \cos ^{2} \vartheta\right)\right) \rrbracket .
\end{aligned}
$$

For the rotational heat flux one obtains

$$
\begin{aligned}
& \sigma\left(\begin{array}{l|l}
1001 \\
1001 & k \\
k
\end{array}\right)_{k l}=\frac{2}{3} \frac{k_{\mathrm{B}}}{c_{k}^{\text {rot }}} \frac{m_{l}}{m_{k}+m_{l}} \llbracket \sigma_{k l}\left(\frac{3}{2}\left(m_{k} / m_{l}\right)\left(\varepsilon_{k}-\varepsilon^{\prime}{ }_{k}\right)^{2}+\gamma^{2}\left(\varepsilon_{k}-\left\langle\varepsilon_{k}\right\rangle_{0}\right)^{2}\right. \\
& \left.+\gamma^{\prime 2}\left(\varepsilon_{k}^{\prime}-\left\langle\varepsilon_{k}\right\rangle_{0}\right)^{2}-2 \gamma \gamma^{\prime} \cos \vartheta\left(\left(\varepsilon_{k}-\left\langle\varepsilon_{k}\right\rangle_{0}\right)\left(\varepsilon_{k}^{\prime}-\left\langle\varepsilon_{k}\right\rangle_{0}\right)\right)\right) \rrbracket \text {, } \\
& \sigma\left(\begin{array}{l|l}
1001 & k \\
1001 & l
\end{array}\right)_{k l}=\frac{2}{3} \frac{k_{\mathrm{B}}}{\sqrt{c_{k}^{\text {rot }} c_{l}^{\text {rot }}}} \frac{\left(m_{k} m_{l}\right)^{1 / 2}}{m_{k}+m_{l}} \llbracket \sigma_{k l}\left(\frac{3}{2}\left(\varepsilon_{k}-\varepsilon_{k}{ }^{\prime}\right)\left(\varepsilon_{l}-\varepsilon_{l}{ }^{\prime}\right)-\gamma^{2}\left(\varepsilon_{k}-\left\langle\varepsilon_{k}\right\rangle_{0}\right)\left(\varepsilon_{l}-\left\langle\varepsilon_{l}\right\rangle_{0}\right)\right. \\
& -\gamma^{\prime 2}\left(\varepsilon_{k}^{\prime}-\left\langle\varepsilon_{k}\right\rangle_{0}\right)\left(\varepsilon_{l}^{\prime}-\left\langle\varepsilon_{l}\right\rangle_{0}\right)+\gamma \gamma^{\prime} \cos \vartheta\left(\left(\varepsilon_{k}-\left\langle\varepsilon_{k}\right\rangle_{0}\right)\left(\varepsilon_{l}^{\prime}-\left\langle\varepsilon_{l}\right\rangle_{0}\right)\right. \\
& \left.\left.+\left(\varepsilon_{k}{ }^{\prime}-\left\langle\varepsilon_{k}\right\rangle_{0}\right)\left(\varepsilon_{l}-\left\langle\varepsilon_{l}\right\rangle_{0}\right)\right)\right) \rrbracket .
\end{aligned}
$$

The coupling cross sections between both heat fluxes are:

$$
\begin{aligned}
\sigma\left(\begin{array}{c|c}
1010 \\
1001
\end{array} \mid \begin{array}{l}
k \\
k
\end{array}\right)_{k l}= & -\frac{4}{3} \sqrt{\frac{2 k_{\mathrm{B}}}{5 c_{k}^{\mathrm{rot}}}}\left(\frac{m_{l}}{m_{k}+m_{l}}\right)^{2} \llbracket \sigma_{k l}\left(\left(5 m_{k} / 2 m_{l}\right)\left(\varepsilon_{k}-\varepsilon_{k}{ }^{\prime}\right)\left(\gamma^{2}-\frac{3}{2}\right)\right. \\
& \left.+\gamma\left(\gamma^{2}-\frac{5}{2}\right)\left(\gamma\left(\varepsilon_{k}-\left\langle\varepsilon_{k}\right\rangle_{0}\right)-\gamma^{\prime}\left(\varepsilon_{k}{ }^{\prime}-\left\langle\varepsilon_{k}\right\rangle_{0}\right) \cos \vartheta\right)\right) \rrbracket, \\
\sigma\left(\begin{array}{c|c}
1010 \\
1001
\end{array} \mid \begin{array}{l}
k \\
l
\end{array}\right)_{k l}= & -\frac{4}{3} \sqrt{\frac{2 k_{\mathrm{B}}}{5 \mathrm{c}_{l}^{\mathrm{rot}}}} \frac{\left(m_{k} m_{l^{3}}\right)^{1 / 2}}{\left(m_{k}+m_{l}\right)^{2}} \llbracket \sigma_{k l}\left(\frac{5}{2}\left(\varepsilon_{l}-\varepsilon_{l}\right)^{\prime}\right)\left(\gamma^{2}-\frac{3}{2}\right) \\
& \left.-\gamma\left(\gamma^{2}-\frac{5}{2}\right)\left(\gamma\left(\varepsilon_{l}-\left\langle\varepsilon_{l}\right\rangle_{0}\right)-\gamma^{\prime}\left(\varepsilon_{l}^{\prime}-\left\langle\varepsilon_{l}\right\rangle_{0}\right) \cos \vartheta\right)\right) \rrbracket,
\end{aligned}
$$

The viscosity cross sections [13] finally are expressed by

$$
\begin{aligned}
\sigma\left(\begin{array}{l|l}
2000 \\
2000
\end{array} \mid \begin{array}{l}
k \\
k
\end{array}\right)_{k l}= & \frac{4}{15}\left(\frac{m_{l}}{m_{k}+m_{l}}\right)^{2} \llbracket \sigma_{k l}\left(3 \gamma^{2} \gamma^{\prime 2}\left(1-\cos ^{2} \vartheta\right)+\left(\Delta \varepsilon_{k l}\right)^{2}\right. \\
& \left.+5\left(m_{k} / m_{l}\right)\left(\gamma^{2}+\gamma^{\prime 2}-2 \gamma \gamma^{\prime} \cos \vartheta\right)\right) \rrbracket, \\
\sigma\left(\begin{array}{l|l}
2000 \\
2000
\end{array} \mid \begin{array}{l}
l \\
l
\end{array}\right)_{k l}= & \frac{4}{15} \frac{m_{k} m_{l}}{\left(m_{k}+m_{l}\right)^{2}} \llbracket \sigma_{k l}\left(3 \gamma^{2} \gamma^{\prime 2}\left(1-\cos ^{2} \vartheta\right)+\left(\Delta \varepsilon_{k l}\right)^{2}\right. \\
& \left.-5\left(\gamma^{2}+\gamma^{\prime 2}-2 \gamma \gamma^{\prime} \cos \vartheta\right)\right) \rrbracket .
\end{aligned}
$$

If, for completeness, from Eqs. (2.19) the translational-rotational-temperature coupling cross sections are listed

$$
\sigma\left(\begin{array}{l|l}
0001 & k \\
0010 & k
\end{array}\right)_{k l}=-\sqrt{\frac{2 k_{\mathrm{B}}}{3 c_{k}^{\mathrm{rot}}}} \frac{m_{l}}{m_{k}+m_{l}} \llbracket \sigma_{k l}\left(\Delta \varepsilon_{k l}\left(\varepsilon_{k}-\varepsilon^{\prime}{ }_{k}\right) \rrbracket=\frac{m_{l}}{m_{k}} \sigma\left(\begin{array}{l}
0001 \\
0010
\end{array}\right)\right.
$$

one can, from Eqs. (2.15) - (2.32), infer the following exact interrelations between effective cross sections:

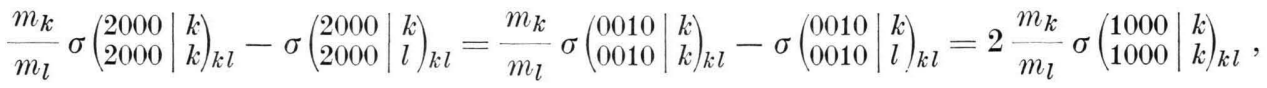

$$
\begin{aligned}
& \sigma\left(\begin{array}{l|l}
1010 & k \\
1001 & k
\end{array}\right)_{k l}+\left(\frac{m_{l}}{m_{k}}\right)^{3 / 2} \sigma\left(\begin{array}{l|l|l}
1010 & l \\
1001 & k
\end{array}\right)_{k l}=\sqrt{\frac{5}{3}} \sigma\left(\begin{array}{l|l|l}
0010 & k \\
0001 & k
\end{array}\right)_{k l}, \\
& \sigma\left(\begin{array}{l|l}
1010 & k \\
1010 & k
\end{array}\right)_{k l}+\left(\frac{m_{l}}{m_{k}}\right)^{3 / 2} \sigma\left(\begin{array}{l|l}
1010 & k \\
1010 & l
\end{array}\right)_{k l}=\frac{4}{3} \sigma\left(\begin{array}{l|l|l}
2000 & k \\
2000 & k
\end{array}\right)_{k l}+\frac{5}{3} \sigma\left(\begin{array}{l|l|l}
0010 & k \\
0010 & k
\end{array}\right)_{k l}-3 \sigma\left(\begin{array}{l}
1000 \\
1000
\end{array} \mid \begin{array}{l}
k \\
k
\end{array}\right)_{k l} \\
& =\frac{4}{3} \frac{m_{l}}{m_{k}} \sigma\left(\begin{array}{l}
2000 \\
2000
\end{array} \mid \begin{array}{l}
k \\
l
\end{array}\right)_{k l}+\frac{5 m_{l}}{3 m_{k}} \sigma\left(\begin{array}{l|l}
0010 & k \\
0010 & l
\end{array}\right)_{k l}+3 \sigma\left(\begin{array}{l}
1000 \\
1000
\end{array} \mid \begin{array}{l}
k \\
k
\end{array}\right)_{k l} .
\end{aligned}
$$

Equations (2.34) and (2.35) have also analogues in the pure gas case [19, 21]. 
Next, effective cross sections characterizing the production of $\left[J_{k}\right]^{[1]}$ and $\left[J_{k}\right]^{[2]}$ polarizations are studied. They determine the magnitudes of the Senftleben-Beenakker effects [13] and of the non-equilibrium polarizations of rotational angular momenta (cf. Ref. [28] for pure gases). These production cross sections are obtained, if in the expression Eq. (2.9) $\Phi^{p q r s}$ is a quantum mechanical tensor $(q \neq 0)$ and $\Phi^{p^{\prime} q^{\prime} r^{\prime} s^{\prime}}$ is a classical one $\left(q^{\prime}=0\right)$. By applying the optical theorem Eq. (1.6) we find

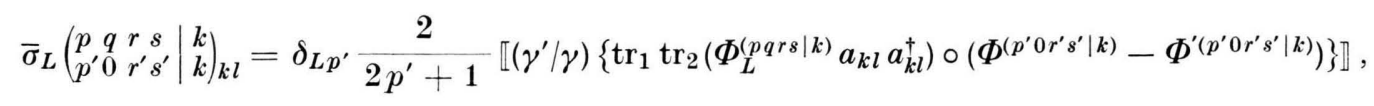

and, using Eq. (2.6),

$$
\sigma\left(\begin{array}{ccc|c}
p & q & r & s \\
p^{\prime} & 0 & r^{\prime} s^{\prime} & k \\
k
\end{array}\right)_{k l} \equiv \sigma_{q}\left(\begin{array}{cccc|c}
p & q & r & s & k \\
p^{\prime} & 0 & r^{\prime} & s^{\prime} & k
\end{array}\right)_{k l}=\left(\frac{2 p^{\prime}+1}{\Omega\left(q, p^{\prime}, p\right)}\right)^{1 / 2} \bar{\sigma}_{p^{\prime}}\left(\begin{array}{lll|l}
p & q & r & s \\
p^{\prime} & 0 & r^{\prime} s^{\prime} & k \\
k
\end{array}\right)_{k l} .
$$

A corresponding expression for the cross section $\bar{\sigma}_{L}\left(\ldots \mid \begin{array}{l}k \\ l\end{array}\right)_{k l}$ is obtained if in Eq. (2.36) $\left(p^{\prime} 0 r^{\prime} s^{\prime} \mid k\right)$ is replaced by $\left(p^{\prime} 0 r^{\prime} s^{\prime} \mid l\right)$. For the following it is useful to introduce molecular orientation cross section tensors of rank $q$ by

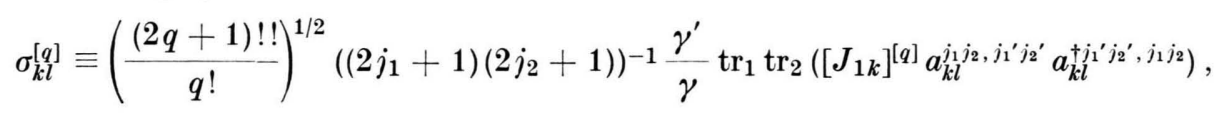

where $\left[J_{1 k}\right]^{[q]}$ is the $q$ th rank irreducible tensor built up from the components of $\boldsymbol{J}_{1 k}$. Obviously, the polarization production cross section tensors (2.38) vanish for a purely spherical molecular interaction.

In ordinary transport processes a $\left[J_{k}\right]^{[1]}$ polarization cannot be produced because of parity reasons. For the production cross section of a $\left[W_{k}\right]^{[1]}\left[J_{k}\right]^{[1]}$ polarization in diffusion or thermal diffusion processes we find

$$
\sigma\left(\begin{array}{l|l}
1100 & k \\
1000 & k
\end{array}\right)_{k l}=-\frac{2}{3} \frac{m_{l}}{m_{k}+m_{l}}\left\langle J_{k^{2}}\right\rangle_{0}^{-1 / 2} \llbracket \sigma_{k l}^{[1]} \circ\left[e^{\prime} e\right]^{[1]} \gamma \gamma^{\prime} \rrbracket
$$

A $\left[W_{k}\right]^{[1]}\left[J_{k}\right]^{[1]}$ polarization can also exist in a heat conducting mixture. The corresponding production cross sections are

and

$$
\sigma\left(\begin{array}{l|l}
1100 & k \\
1010 & k
\end{array}\right)_{k l}=\frac{-4}{3 \sqrt{10}}\left(\frac{m_{l}}{m_{k}+m_{l}}\right)^{2}\left\langle J_{\left.k^{2}\right\rangle_{0}-1 / 2} \llbracket \sigma_{k l}^{[1]} \circ\left[e^{\prime} e\right]^{[1]} \gamma \gamma^{\prime}\left(\gamma^{\prime 2}-\frac{5}{2}\right) \rrbracket,\right.
$$

$$
\sigma\left(\begin{array}{l|l}
1100 & k \\
1010 & l
\end{array}\right)_{k l}=+\frac{4}{3 \sqrt{10}} \frac{m_{k}^{3 / 2} m_{l}^{1 / 2}}{\left(m_{k}+m_{l}\right)^{2}}\left\langle J_{k^{2}}\right\rangle_{0}-1 / 2 \llbracket \sigma_{k l}^{[1]} \circ\left[e^{\prime} e\right]^{[1]} \gamma \gamma^{\prime}\left(\gamma^{\prime 2}-\frac{5}{2}\right) \rrbracket,
$$

as well as

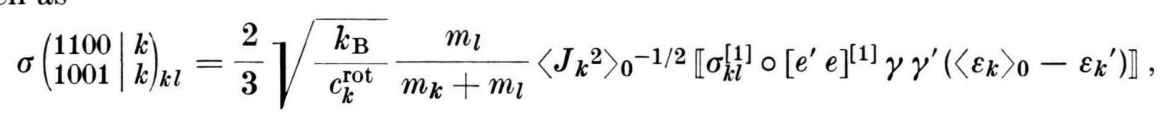

and

$$
\sigma\left(\begin{array}{l|l}
1100 & k \\
1001 & l
\end{array}\right)_{k l}=\frac{2}{3} \sqrt{\frac{k_{\mathrm{B}}}{c_{l}^{\text {rot }}}} \frac{\left(m_{k} m_{l}\right)^{1 / 2}}{m_{k}+m_{l}}\left\langle J_{k^{2}}\right\rangle_{0}-1 / 2 \llbracket \sigma_{k l}^{[1]} \circ\left[e^{\prime} e\right]^{[1]} \gamma \gamma^{\prime}\left(\varepsilon_{l}^{\prime}-\left\langle\varepsilon_{l}\right\rangle_{0}\right) \rrbracket .
$$

In the expressions Eqs. $(2.39)-(2.43),\left[e^{\prime} e\right]^{[1]}$ denotes the vector built up by $\boldsymbol{e}^{\prime}$ and $\boldsymbol{e}$, viz. the cross product $\boldsymbol{e}^{\prime} \times \boldsymbol{e}$ which is normal to the plane of scattering; $\left[e^{\prime} e\right]^{[2]}$ is then a 2 nd rank tensor $\left(=\widetilde{\boldsymbol{e}^{\prime} \boldsymbol{e}}\right)$.

More important from an experimental point of view $[1,2,4 \mathrm{a}]$ is the Kagan polarization $\left[W_{k}\right]^{[1]}\left[J_{k}\right]^{[2]}$. For its production in a diffusion experiment e.g. one obtains

$$
\sigma\left(\begin{array}{c|c}
1200 & k \\
1000 & k
\end{array}\right)_{k l}=\frac{4}{5}\left\langle J_{k}^{2}\left(J_{k}^{2}-\frac{3}{4}\right)\right\rangle_{0}-1 / 2 \frac{m_{l}}{m_{k}+m_{l}} \llbracket \sigma_{k l}^{[2]} \circ\left(\gamma^{2}[e]^{[2]}-\gamma \gamma^{\prime}\left[e^{\prime} e\right]^{[2]}\right) \rrbracket .
$$


For the corresponding cross sections describing the production of a Kagan-polarization by the heat fluxes we find

and

$$
\begin{aligned}
\sigma\left(\begin{array}{c|c}
1200 \\
1010
\end{array} \mid \begin{array}{l}
k \\
k
\end{array}\right)_{k l}= & -\frac{4}{5} \sqrt{\frac{2}{5}}\left(\frac{m_{l}}{m_{k}+m_{l}}\right)^{2}\left\langle J_{k}^{2}\left(J_{k}^{2}-\frac{3}{4}\right)\right\rangle_{0}^{-1 / 2} \llbracket \sigma_{k l}^{[2]} \circ\left(\gamma^{2}\left(1+\left(m_{k} / m_{l}\right)+\left(\gamma^{2}-\frac{7}{2}\right)\right)[e]^{[2]}\right. \\
& \left.-\left(m_{k} / m_{l}\right) \gamma^{\prime 2}\left[e^{\prime}\right]^{[2]}+\gamma \gamma^{\prime}\left(\frac{5}{2}-\gamma^{\prime 2}\right)\left[e^{\prime} e\right]^{[2]}\right) \rrbracket
\end{aligned}
$$

$$
\begin{aligned}
\sigma\left(\begin{array}{c|c}
1200 \\
1010
\end{array} \mid \begin{array}{l}
k \\
l
\end{array}\right)_{k l}= & -\frac{4}{5} \sqrt{\frac{2}{5}} \frac{\left(m_{k}^{3} m_{l}\right)^{1 / 2}}{\left(m_{k}+m_{l}\right)^{2}}\left\langle J_{k}{ }^{2}\left(J_{k}{ }^{2}-\frac{3}{4}\right)\right\rangle_{0}-1 / 2 \llbracket \sigma_{k l}^{[2]} \circ\left(\gamma^{2}\left(\frac{7}{2}-\gamma^{2}\right)[e]^{[2]}-\gamma^{\prime 2}\left[e^{\prime}\right]^{[2]}\right. \\
& \left.-\gamma \gamma^{\prime}\left(\frac{5}{2}-\gamma^{\prime 2}\right)\left[e^{\prime} e\right]^{[2]}\right) \rrbracket,
\end{aligned}
$$

as well as

$$
\begin{aligned}
\sigma\left(\begin{array}{c|c}
1200 \\
1001
\end{array} \mid \begin{array}{l}
k \\
k
\end{array}\right)_{k l}= & \frac{4}{5} \sqrt{\frac{k_{\mathrm{B}}}{c_{k}^{\text {rot }}}} \frac{m_{l}}{m_{k}+m_{l}}\left\langle J_{k^{2}}\left(J_{k}{ }^{2}-\frac{3}{4}\right)\right\rangle_{0}-1 / 2 \llbracket \sigma_{k l}^{[2]} \circ\left(\gamma^{2}\left(\varepsilon_{k}-\left\langle\varepsilon_{k}\right\rangle_{0}\right)[e]^{[2]}\right. \\
& \left.-\gamma \gamma^{\prime}\left(\varepsilon_{k}{ }^{\prime}-\left\langle\varepsilon_{k}\right\rangle_{0}\right)\left[e^{\prime} e\right]^{[2]}\right) \rrbracket,
\end{aligned}
$$

and

$$
\begin{aligned}
\sigma\left(\begin{array}{c|c}
1200 \\
1001
\end{array} \mid \begin{array}{l}
k \\
l
\end{array}\right)_{k l}= & \frac{4}{5} \sqrt{\frac{k_{\mathrm{B}}}{c_{l}^{\text {rot }}}} \frac{\left(m_{k} m_{l}\right)^{1 / 2}}{m_{k}+m_{l}}\left\langle J_{k}{ }^{2}\left(J_{k}{ }^{2}-\frac{3}{4}\right)\right\rangle_{0}-1 / 2 \llbracket \sigma_{k l}^{[2]} \circ\left(\gamma^{2}\left(\left\langle\varepsilon_{l}\right\rangle_{0}-\varepsilon_{l}\right)[e]^{[2]}\right. \\
& \left.+\gamma \gamma^{\prime}\left(\varepsilon^{\prime}{ }_{l}-\left\langle\varepsilon_{l}\right\rangle_{0}\right)\left[e^{\prime} e\right]^{[2]}\right) \rrbracket .
\end{aligned}
$$

The production cross sections for a tensor polarization $\left[J_{k}\right]^{[2]}$ in a streaming gas mixture are given by

$$
\sigma\left(\begin{array}{l|l}
0200 \\
2000 & k \\
k
\end{array}\right)_{k l}=\frac{2 \sqrt{2}}{5} \frac{m_{l}}{m_{k}+m_{l}}\left\langle J_{k}^{2}\left(J_{k}^{2}-\frac{3}{4}\right)\right\rangle_{0}-1 / 2 \llbracket \sigma_{k l}^{[2]} \circ\left(\gamma^{2}[e]^{[2]}-\gamma^{\prime 2}\left[e^{\prime}\right]^{[2]}\right) \rrbracket,
$$

and

$$
\sigma\left(\begin{array}{l|l}
0200 & k \\
2000 & l
\end{array}\right)_{k l}=\frac{2 \sqrt{2}}{5} \frac{m_{k}}{m_{k}+m_{l}}\left\langle J_{k}{ }^{2}\left(J_{k}^{2}-\frac{3}{4}\right)\right\rangle_{0}-1 / 2 \llbracket \sigma_{k l}^{[2]} \circ\left(\gamma^{2}[e]^{[2]}-\gamma^{\prime 2}\left[e^{\prime}\right]^{[2]}\right) \rrbracket .
$$

Also a $\left[W_{k}\right]^{[2]} J_{k}{ }^{[1]}$ polarization can be produced in a streaming gas mixture. The corresponding production cross sections are

$$
\left.\sigma\left(\begin{array}{l|l}
2100 \\
2000 & k \\
k
\end{array}\right)_{k l}=-\frac{8}{15}\left(\frac{m_{l}}{m_{k}+m_{l}}\right)^{2}\left\langle J_{k^{2}}\right\rangle_{0}^{-1 / 2} \llbracket \sigma_{k l}^{[1]} \circ\left[e^{\prime} e\right]^{[1]} \gamma \gamma^{\prime}\left(\gamma \gamma^{\prime} \cos \vartheta+\frac{5}{2}\left(m_{k} / m_{l}\right)\right)\right],
$$

and

$$
\sigma\left(\begin{array}{l|l}
2100 \\
2000
\end{array} \mid \begin{array}{l}
l \\
l
\end{array}\right)_{k l}=-\frac{8}{15} \frac{m_{l} m_{k}}{\left(m_{k}+m_{l}\right)^{2}}\left\langle J_{k^{2}}\right\rangle_{0}-1 / 2 \llbracket \sigma_{k l}^{[1]} \circ\left[e^{\prime} e\right]^{[1]} \gamma \gamma^{\prime}\left(\gamma \gamma^{\prime} \cos \vartheta-\frac{5}{2}\right) \rrbracket .
$$

From inspection of Eqs. (2.39) - (2.52) the following non-trivial exact relations (the trivial exact relations due to momentum conservation (2.21) are not listed here) among production cross sections can be inferred:

$$
\begin{aligned}
& \sigma\left(\begin{array}{l|l}
1100 & k \\
1010 & l
\end{array}\right)_{k l}=-\left(\frac{m_{k}}{m_{l}}\right)^{3 / 2} \sigma\left(\begin{array}{l|l|l}
1100 & k \\
1010 & k
\end{array}\right)_{k l}, \\
& \sigma\left(\begin{array}{l|l}
0200 \\
2000
\end{array} \mid \begin{array}{l}
l \\
l
\end{array}\right)_{k l}=\frac{m_{k}}{m_{l}} \sigma\left(\begin{array}{l|l|l}
0200 & k \\
2000 & k
\end{array}\right)_{k l}, \\
& \sigma\left(\begin{array}{l|l}
1200 & k \\
1010 & k
\end{array}\right)_{k l}+\left(\frac{m_{l}}{m_{k}}\right)^{3 / 2} \sigma\left(\begin{array}{l|l|l}
1200 & k \\
1010 & l
\end{array}\right)_{k l}=-\frac{2}{\sqrt{5}} \sigma\left(\begin{array}{l}
0200 \\
2000
\end{array} \mid \begin{array}{l}
k \\
k
\end{array}\right)_{k l},
\end{aligned}
$$

and, finally,

$$
\sigma\left(\begin{array}{l|l}
2100 & k \\
2000 & k
\end{array}\right)_{k l}-\frac{m_{l}}{m_{k}} \sigma\left(\begin{array}{l|l}
2100 & k \\
2000 & l
\end{array}\right)_{k l}=2 \sigma\left(\begin{array}{l|l}
1100 & k \\
1000 & k
\end{array}\right)_{k l} .
$$


The last group of effective cross sections to be discussed are the relaxation cross sections for quantum mechanical ( $\boldsymbol{J}$-dependent) expansion tensors, i.e. for rotational angular momentum polarizations. Cross sections describing the coupling between different $\boldsymbol{J}$-polarizations are disregarded here since there have not been observed any phenomena where they could play a role.

A general expression for the relaxation cross sections of angular momentum polarizations is obtained from Eq. (2.9) by use of the optical theorem Eq. (1.10):

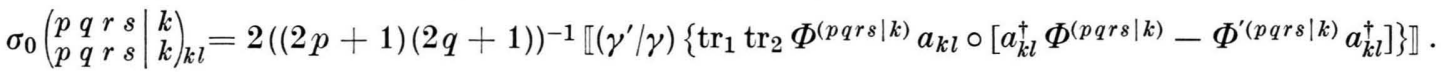

Here, the dot $\circ$ denotes a separate contraction over tensor indices belonging to $p$ and to $q$. The expression for $\sigma_{0}\left(\begin{array}{ccc|c}p & q & r & s \\ p & q & r & s\end{array} \mid \begin{array}{l}l \\ l\end{array}\right)_{k l}$ is obtained, if in Eq. (2.57) in the second bracket (pqrs|k) is replaced by $(p q r s \mid l)$. It should be mentioned, that for $[W]^{[p]}[J]^{[q]}$ polarizations not only $\sigma_{0}(\cdots)$ exists but in general terms $\sigma_{K}(\cdots)$ appear where $0 \leqq K \leqq \min (2 p, 2 q)$, cf. Equation (2.1). Experiments, however, have shown that only the cross sections with $K=0$ are important, the others being small, i.e. of the same order as the coupling cross sections of two different $\boldsymbol{J}$-polarizations.

For the further treatment it is useful to introduce molecular reorientation cross sections by

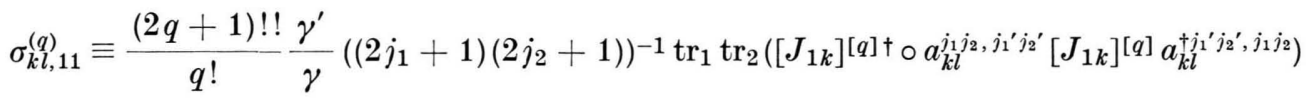

and

$$
\sigma_{k l, 12}^{(q)} \equiv \frac{(2 q+1) ! !}{q !} \frac{\gamma^{\prime}}{\gamma}\left(\left(2 j_{1}+1\right)\left(2 j_{2}+1\right)\right)^{-1} \operatorname{tr}_{1} \operatorname{tr}_{2}\left(\left[J_{1 k}\right]^{[q] \dagger} \circ a_{k l}^{j_{1} j_{2}, j_{1} j_{j_{2}}}\left[J_{2 l}\right]^{[q]} a_{k l}^{\dagger j_{1}^{\prime} j_{2}, j_{1} j_{2}}\right) .
$$

A further cross section $\tilde{\sigma}_{k l, 12}^{(q)}$ is defined by interchange of $a_{k l}$ and $\left[J_{2 l}\right]^{[q]}$ in $(2.59)$. While the second reorientation cross sections vanish in the case of a purely spherical molecular interaction, the first ones yield: ( $\left|a_{k l}^{(0)}\right|^{2}$ being the ordinary differential cross section due to a spherical potential)

$$
\operatorname{sph} \sigma_{k l, 11}^{(q)}=g_{1}^{(q)}\left|a_{k l}^{(0)}\right|^{2}, \quad g_{m}^{(q)} \equiv \operatorname{tr}_{1} \operatorname{tr}_{2} \frac{\left[J_{m}\right]^{[q] \dagger} \circ\left[J_{m}\right]^{[q]}}{\left(2 j_{1}+1\right)\left(2 j_{2}+1\right)}, \quad m=1,2 .
$$

Next, the bracket symbol expressions for effective relaxation cross sections of pure $\boldsymbol{J}$-polarizations, namely vector- and tensor polarization are considered:

$$
\begin{aligned}
& \sigma\left(\begin{array}{l|l}
0100 & k \\
0100 & k
\end{array}\right)_{k l}=\frac{2}{3}\left\langle J_{k^{2}}\right\rangle_{0}^{-1} \llbracket \sigma_{k l, 11}^{(1)}-\sigma_{k l} g_{1}^{(1)} \rrbracket, \\
& \sigma\left(\begin{array}{l|l}
0100 & k \\
0100 & l
\end{array}\right)_{k l}=\frac{2}{3}\left\langle J_{k}^{2}\right\rangle_{0}^{-1 / 2}\left\langle J_{l^{2}}\right\rangle_{0}-1 / 2 \llbracket \sigma_{k l, 12}^{(1)}-\tilde{\sigma}_{k l, 12}^{(1)} \rrbracket, \\
& \sigma\left(\begin{array}{c|c}
0200 & k \\
0200 & k
\end{array}\right)_{k l}=\frac{2}{5}\left\langle J_{k}^{2}\left(J_{k}^{2}-\frac{3}{4}\right)\right\rangle_{0}-1 \llbracket \sigma_{k l, 11}^{(2)}-\sigma_{k l} g_{1}^{(2) \rrbracket}, \\
& \sigma\left(\begin{array}{l|l}
0200 \\
0200 & l
\end{array}\right)_{k l}=\frac{2}{5}\left\langle J_{k}^{2}\left(J_{k}^{2}-\frac{3}{4}\right)\right\rangle_{0}-1 / 2\left\langle J_{l}^{2}\left(J_{l}^{2}-\frac{3}{4}\right)\right\rangle_{0}-1 / 2 \llbracket \sigma_{k l, 12}^{(2)}-\tilde{\sigma}_{k l, 12}^{(2)} \rrbracket .
\end{aligned}
$$

It is clear that the cross sections (2.61)-(2.64) vanish for a purely spherical interaction. If the scattering amplitude $a_{k l}$ is expanded in powers of the nonspherical part of the interaction, they are quadratic in the nonsphericity.

The last group of relaxation cross sections to be considered are the quantities $\sigma_{0}(\cdots)$ connected with (1100), (1200), and (2100) polarizations, respectively. Here, one obtains

$$
\begin{aligned}
& \sigma_{0}\left(\begin{array}{l|l}
1100 \\
1100
\end{array} \mid \begin{array}{l}
k \\
k
\end{array}\right)_{k l}=\frac{2}{3} \frac{m_{k}}{m_{k}+m_{l}}\left\langle J_{k}\right\rangle_{0}{ }^{-1} \llbracket \sigma_{k l} g_{1}^{(1)}\left(1+\left(2 m_{l} / 3 m_{k}\right) \gamma^{2}\right) \\
& -\sigma_{k l, 11}^{(1)}\left(1+\left(2 m_{l} / 3 m_{k}\right) \gamma \gamma^{\prime} \cos \vartheta\right) \rrbracket, \\
& \sigma_{0}\left(\begin{array}{l|l}
1100 & k \\
1100 & l
\end{array}\right)_{k l}=\frac{2}{3} \frac{\left(m_{k} m_{l}\right)^{1 / 2}}{m_{k}+m_{l}}\left\langle J_{k^{2}}\right\rangle_{0}-1 / 2\left\langle J_{l}^{2}\right\rangle_{0}-1 / 2 \llbracket \tilde{\sigma}_{k l, 12}^{(1)}\left(1-\frac{2}{3} \gamma^{2}\right) \\
& -\sigma_{k l, 12}^{(1)}\left(1-\frac{2}{3} \gamma \gamma^{\prime} \cos \vartheta\right) \rrbracket
\end{aligned}
$$


and finally

$$
\begin{aligned}
\sigma_{0}\left(\begin{array}{l}
1209 \\
1200
\end{array} \mid \begin{array}{l}
k \\
k
\end{array}\right)_{k l}= & \frac{2}{5} \frac{m_{k}}{m_{k}+m_{l}}\left\langle J_{k}{ }^{2}\left(J_{k^{2}}-\frac{3}{4}\right)\right\rangle_{0}-1 \llbracket \sigma_{k l} g_{1}^{(2)}\left(1+\left(2 m_{l} / 3 m_{k}\right) \gamma^{2}\right) \\
& -\sigma_{k l, 11}^{(2)}\left(1+\left(2 m_{l} / 3 m_{k}\right) \gamma \gamma^{\prime} \cos \vartheta\right) \rrbracket, \\
\sigma_{0}\left(\begin{array}{l}
1200 \mid \\
1200
\end{array} \mid \begin{array}{l}
k \\
l
\end{array}\right)_{k l}= & \frac{2}{5} \frac{\left(m_{k} m_{l}\right)^{1 / 2}}{m_{k}+m_{l}}\left\langle J_{k}^{2}\left(J_{k^{2}}{ }^{2}-\frac{3}{4}\right)\right\rangle_{0}-1 / 2\left\langle J_{l}^{2}\left(J_{l}^{2}-\frac{3}{4}\right)\right\rangle_{0}-1 / 2 \llbracket \tilde{\sigma}_{k l, 12}^{(2)}\left(1-\frac{2}{3} \gamma^{2}\right) \\
& -\sigma_{k l, 12}^{(2)}\left(1-\frac{2}{3} \gamma \gamma^{\prime} \cos \vartheta\right) \rrbracket,
\end{aligned}
$$

$$
\begin{aligned}
& \sigma_{0}\left(\begin{array}{l|l}
2100 \\
2100
\end{array} \mid \begin{array}{l}
k \\
k
\end{array}\right)_{k l}=\frac{4}{15}\left(\frac{m_{k}}{m_{k}+m_{l}}\right)^{2}\left\langle J_{k^{2}}\right\rangle_{0}^{-1} \llbracket \sigma_{k l} g_{1}^{(1)}\left(\begin{array}{l}
5 \\
2
\end{array}+\left(10 m_{l} / 3 m_{k}\right) \gamma^{2}+\frac{2}{3}\left(m_{l} / m_{k}\right)^{2} \gamma^{4}\right) \\
& -\sigma_{k l, 11}^{(2)}\left({ }_{2}^{5}+\left(10 m_{l} / 3 m_{k}\right) \gamma \gamma^{\prime} \cos \vartheta+\frac{1}{3}\left(m_{l} / m_{k}\right)^{2} \gamma^{2} \gamma^{\prime 2}\left(3 \cos ^{2} \vartheta-1\right)\right) \rrbracket, \\
& \sigma_{0}\left(\begin{array}{l|l}
2100 \\
2100
\end{array} \mid \begin{array}{l}
k \\
l
\end{array}\right)_{k l}=\frac{4}{15} \frac{m_{k} m_{l}}{\left(m_{k}+m_{l}\right)^{2}}\left\langle J_{k}\right\rangle_{0}^{-1 / 2}\left\langle J_{l}^{2}\right\rangle_{0}-1 / 2 \llbracket \tilde{\sigma}_{k l, 12}^{(1)}\left({ }_{2}^{5}-{ }_{3}^{10} \gamma^{2}+\frac{2}{3} \gamma^{4}\right) \\
& \left.-\sigma_{k l, 12}^{(1)}\left({ }_{2}^{5}-{ }_{3}^{10} \gamma \gamma^{\prime} \cos \vartheta+\frac{1}{3} \gamma^{2} \gamma^{\prime 2}\left(3 \cos ^{2} \vartheta-1\right)\right)\right] \text {. }
\end{aligned}
$$

\section{Approximate Relations}

Approximate relations between effective cross sections are obtained if an approximation for the molecular scattering amplitude is made.

The simplest approximation consists in neglecting the nonspherical part of the intermolecular potential at all. This crude approximation can certainly not be made for relaxation cross sections of $\boldsymbol{J}$-polarizations and for coupling cross sections of $\boldsymbol{J}$-dependent and $\boldsymbol{J}$-independent quantities since those would all be equal to zero. For relaxation cross sections of classical moments, howeve', or of $[\boldsymbol{W}]^{p}[\boldsymbol{J}]^{q}$ correlations this àpproximation might be a first step yielding not too bad results for molecules with small nonsphericity $\left(\mathrm{H}_{2}, \mathrm{D}_{2}\right)$. With the help of Eq. (2.60) these "spherical" cross sections can furthermore be expressed in terms of well-known Chapman-cowling collision integrals [23] defined by

$$
\Omega_{k l}^{(m, n)}=\frac{v_{k l}}{4} 2 \pi \int_{0}^{\infty} e^{-\gamma^{2}} \gamma^{2 n+3} \int_{0}^{\pi}\left(1-\cos ^{m} \vartheta\right) \sigma_{k l}^{(0)} \sin \vartheta \mathrm{d} \vartheta \mathrm{d} \gamma,
$$

Here, $\sigma_{k l}^{(0)}=\left|a_{k l}^{(0)}\right|^{2}$ is the differential cross section calculated with the scattering amplitude $a_{k l}^{(0)}$ pertaining to the spherical potential. In this way the following relations for relaxation cross sections and coupling cross sections of classical moments are obtained:

$$
\begin{aligned}
& \operatorname{sph} \sigma\left(\begin{array}{c|c}
0010 & k \\
0010 & k
\end{array}\right)_{k l}=-\operatorname{sph} \sigma\left(\begin{array}{c|c}
0010 & k \\
0010 & l
\end{array}\right)_{k l}=v_{k l}^{-1} \frac{32}{3} \frac{m_{k} m_{l}}{\left(m_{k}+m_{l}\right)^{2}} \Omega^{(1,1)}, \\
& \sigma_{\mathrm{sph}}\left(\begin{array}{c|c}
1000 & k \\
1000 & k
\end{array}\right)_{k l}=v_{k l}^{-1} \frac{32}{3} \frac{m_{l}}{m_{k}+m_{l}} \Omega_{k l}^{(1,1)}=\frac{m_{k}+m_{l}}{2 m_{k}} \operatorname{sph} \sigma\left(\begin{array}{c|c|c}
0010 & k \\
0010 & k
\end{array}\right)_{k l}, \\
& \operatorname{sph} \sigma\left(\begin{array}{l|l}
1010 \\
1010
\end{array} \mid \begin{array}{l}
k \\
k
\end{array}\right)_{k l}=v_{k l}^{-1} \frac{m_{k}^{2} m_{l}}{\left(m_{k}+m_{l}\right)^{3}}\left[\left(16+\frac{40}{3}\left(\frac{m_{l}}{m_{k}}\right)^{2}\right) \Omega_{k l}^{(1,1)}-\frac{32}{3}\left(\frac{m_{l}}{m_{k}}\right)^{2} \Omega_{k l}^{(1,2)}\right. \\
& \left.+\frac{32}{15}\left(\frac{m_{l}}{m_{k}}\right)^{2} \Omega_{k l}^{(1,3)}+\frac{64}{15} \frac{m_{l}}{m_{k}} \Omega_{k l}^{(2,2)}\right] \text {, } \\
& \operatorname{sph} \sigma\left(\begin{array}{l}
1010 \\
1010
\end{array} \mid \begin{array}{l}
l \\
l
\end{array}\right)_{k l}=v_{k l}^{-1} \frac{\left(m_{k} m_{l}\right)^{3 / 2}}{\left(m_{k}+m_{l}\right)^{3}}\left[-\frac{88}{3} \Omega_{k l}^{(1,1)}+\frac{32}{3} \Omega_{k l}^{(1,2)}-\frac{32}{15} \Omega_{k l}^{(1,3)}+\frac{64}{15} \Omega_{k l}^{(2,2)}\right] \text {, } \\
& \operatorname{sph} \sigma\left(\begin{array}{l|l}
1000 \\
1010
\end{array} \mid \begin{array}{l}
k \\
k
\end{array}\right)_{k l}=-v_{k l}^{-1} \frac{16}{3} \sqrt{\frac{2}{5}}\left(\frac{m_{l}}{m_{k}+m_{l}}\right)^{2}\left[\left[\Omega_{k l}^{(1,2)}-\frac{5}{2} \Omega_{k l}^{(1,1)}\right],\right.
\end{aligned}
$$




$$
\begin{aligned}
& \operatorname{sph} \sigma\left(\begin{array}{l|l}
2000 & k \\
2000 & k
\end{array}\right)_{k l}=v_{k l}^{-1} \frac{16}{15} \frac{m_{k} m_{l}}{\left(m_{k}+m_{l}\right)^{2}}\left[10 \Omega_{k l}^{(1,1)}+3 \frac{m_{l}}{m_{k}} \Omega_{k l}^{(2,2)}\right], \\
& \operatorname{sph} \sigma\left(\begin{array}{l|l}
2000 & k \\
2000 & l
\end{array}\right)_{k l}=v_{k l}^{-1} \frac{16}{15} \frac{m_{k} m_{l}}{\left(m_{k}+m_{l}\right)^{2}}\left[-10 \Omega_{k l}^{(1,1)}+3 \Omega_{k l}^{(2,2)}\right]
\end{aligned}
$$

Equations (3.2) - (3.8) are in agreement with well-known results of the literature [29].

For the relaxation cross section of the rotational heat flux one finds

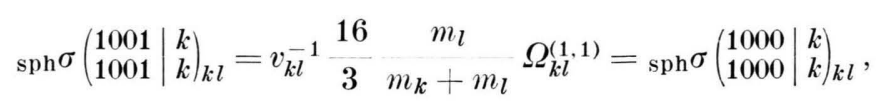

which means that in this approximation only diffusion of rotational energy takes place. The corresponding cross section $\operatorname{sph} \sigma\left(\begin{array}{c}1001 \\ \mathbf{1 0 0 1}\end{array} \mid \begin{array}{l}k \\ l\end{array}\right)_{k l}$ vanishes. The relaxation cross sections for the various types of $[W]^{p}[J]^{q}$ polarizations considered are

$$
\begin{aligned}
& \operatorname{sph} \sigma\left(\begin{array}{l|l|l}
1100 & k \\
1100 & k
\end{array}\right)_{k l}=\operatorname{sph} \sigma\left(\begin{array}{l|l|l}
1200 & k \\
1200 & k
\end{array}\right)_{k l}=\operatorname{sph} \sigma\left(\begin{array}{l}
1000 \\
1000
\end{array} \mid k\right)_{k l}, \\
& \operatorname{sph} \sigma\left(\begin{array}{l|l}
2100 & k \\
2100 & k
\end{array}\right)_{k l}=\operatorname{sph} \sigma\left(\begin{array}{l|l}
2000 & k \\
2000 & k
\end{array}\right)_{k l},
\end{aligned}
$$

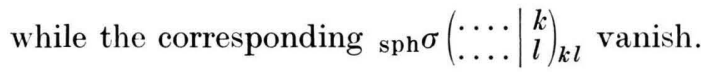

The next step, appropriate for the approximate evaluation of polarization production cross sections consists in taking into account the nonspherical part of the potential in the cross sections in a linear way (elastic DWBA [17, 30]). If the DWBA-scattering amplitude [19] possesses an elastic part, e.g. due to a $\mathrm{P}_{2}$-interaction, there exists also an elastic part of the production cross sections linear in the nonsphericity due to an interference term of the "spherical" and "nonspherical" part of the scattering amplitude. This elastic "nonspherical" part of the scattering matrix factorizes into an internal ( $\boldsymbol{J}$-dependent) and a purely translational part (cf. Refs. [17, 30]). As a consequence, for molecules with a dominant $\mathrm{P}_{2}$-term in their nonspherical interaction (e.g. homonuclear diatomics) one has for arbitrary functions $G_{k}\left(J_{1}^{2}\right)$ :

$$
\begin{gathered}
\left(Z_{k}^{\mathrm{rot}} Z_{l}^{\mathrm{rot}}\right)^{-1} \sum_{j_{1}, j_{2}} G_{k}\left(J_{1}^{2}\right) \operatorname{tr}_{1} \operatorname{tr}_{2}\left(a_{k l} a_{k l}^{\dagger} \overline{\boldsymbol{J}_{1 k} \boldsymbol{J}_{1 k}}\right) \exp \left(-\varepsilon_{k}\left(j_{1}\right)-\varepsilon_{l}\left(j_{2}\right)\right) \\
\left.\left.=\left\langle G_{k}\left(J_{1}^{2}\right) J_{1}^{2}\right\rangle_{0}\left(F_{1}(\gamma) \overline{(\boldsymbol{e} \boldsymbol{e}}+\overline{\boldsymbol{e}^{\prime} \boldsymbol{e}^{\prime}}\right)+F_{2}(\gamma) \overline{\boldsymbol{e} \boldsymbol{e}^{\prime}}\right)\right)
\end{gathered}
$$

Here, $F_{1}(\gamma)$ and $F_{2}(\gamma)$ are scalar functions built up from the phase shifts pertaining to the spherical potential and the radial integrals involving the radial part of the $\mathrm{P}_{2}$-term and the partial distorted waves. For details see [30]. For the derivation of approximate relations between tensor polarization production cross sections only the factorization property expressed by (3.12) is important. As an immediate consequence of the symmetry of the r.h.s. of Eq. (3.12) in $\boldsymbol{e}$ and $\boldsymbol{e}^{\prime}$, one has in the elastic DWBA

$$
{ }_{\mathrm{el}} \sigma_{k l}^{[2]} \circ \overline{\boldsymbol{e} \boldsymbol{e}}={ }_{\mathrm{el}} \sigma_{k l}^{[2]} \circ \overline{\boldsymbol{e}^{\prime} \boldsymbol{e}^{\prime}} \neq \mathrm{el}_{k l}^{[2]} \circ \overline{\boldsymbol{e} \boldsymbol{e}^{\prime}} .
$$

From Eqs. (2.45), (2.46) and (3.13) we can immediately infer the approximate relation (also obtainable from Eq. (2.55) by neglecting inelastic collisions)

$$
\mathrm{el} \sigma\left(\begin{array}{l|l}
1200 & k \\
1010 & l
\end{array}\right)_{k l}=-\left(\frac{m_{k}}{m_{l}}\right)^{3 / 2} \text { el } \sigma\left(\begin{array}{l|l|l}
1200 & k \\
1010 & k
\end{array}\right)_{k l} .
$$

The factorization property (3.12) with $G_{k}\left(J_{1}^{2}\right)=\varepsilon_{k}\left(J_{1}^{2}\right)-\left\langle\varepsilon_{k}\right\rangle_{0}$ enables us to compare the (1200)-(1000) and (1200)-(1001) coupling cross sections (Eqs. (2.44) and (2.47)) in this elastic approximation with the result

$$
\mathrm{el} \sigma\left(\begin{array}{l|l}
1200 & k \\
1001 & k
\end{array}\right)_{k l}=\left\langle\varepsilon_{k}\right\rangle_{0}^{-1}\left(c_{k}^{\mathrm{rot}} / k_{\mathrm{B}}\right)^{1 / 2} \mathrm{el} \sigma\left(\begin{array}{c|c|c}
1200 & k \\
1000 & k
\end{array}\right)_{k l}
$$


This relation has been used by 't Hooft in analyzing the data on the magnetic field effect on thermal conductivity [1, 4a]. One should keep in mind, however, that the validity of Eqs. (3.14), (3.15) is guaranteed only for molecules with essentially small nonsphericity, i.e. for mixtures of $\mathrm{H}_{2}, \mathrm{D}_{2}$ with noble gas atoms and might be questionable for heavier molecules $\left(\mathrm{N}_{2}\right)$. Because of Eq. (1.13), the relaxation cross sections of vector and tensor polarization are at least quadratic in the nonsphericity as it is also true for pure gases [17].

Finally, it should be stressed that in contrast to relations between collision cross sections for pure gases obtained by Moraal and Snider [22] in plane-wave cutoff-Born approximation plus high temperature limit, the approximate relations given here are based on DWBA and independent of temperature.

\section{Acknowledgement}

The authors thank Prof. Dr. H. F. P. Knaap for valuable discussions.

This work is part of the research program of the "Stichting voor Fundamenteel Onderzoek der Materie (FOM)" and has been made possible by financial support from the "Nederlandse Organisatie voor Zuiver-Wetenschappelijk Onderzoek (ZWO)“،.

[1] J. P. J. Heemskerk, G. F. Bulsing, and H. F. P. Knaap, Physica 71, 515 (1974).

[2] S. Bhanja, Udayan De, and A. K. Barua, Physica $90 \mathrm{~A}, 167$ (1978).

[3] A. L. J. Burgmans, P. G. van Ditzhuyzen, and H. F. P. Knaap, Z. Naturforsch. 28a, 849 (1973).

[4] G. E. J. Eggermont, P. Oudeman and L. J. F. Hermans, Phys. Letters 50 A, 173 (1974); E. Mazur, G. W.'t Hooft, and L. J. F. Hermans, Phys. Letters $64 \mathrm{~A}, 35$ (1977).

[4a] G. W.'t Hooft et al., Physica, 98 A, 41 (1979).

[5] R. A. Keijser, K. D. van den Hout, and H. F. P. Knaap, Physica 76, 577 (1974).

[6] F. Baas, J. N. Breunese, and H. F. P. Knaap, Physica $88 \mathrm{~A}, 34$ (1977).

[7] L. Waldmann, Z. Naturforsch. 12a, 660 (1957); 13a, 609 (1958); R. F. Snider, J. Chem. Phys. 32, 1051 (1960).

[8] H. H. Raum and W. E. Köhler, Z. Naturforsch. 25a, $1178(1970)$.

[9] W. E. Köhler and H. H. Raum, Z. Naturforsch. 27 a, 1383 (1972).

[10] W. E. Köhler and J. Halbritter, Z. Naturforsch. 30a, 1114 (1975).

[11] W. E. Köhler and J. Halbritter, Physica 74, 294 (1974).

[12] J. Halbritter and W. E. Köhler, Physica 76, 224 (1974).

[13] G. E. J. Eggermont, H. Vestner, and H. F. P. Knaap, Physica 82 A, 23 (1976).

[14] W. E. Köhler and G. E. J. Eggermont, Physica 91 A, 17 (1978).
[15] W. E. Köhler, S. Hess, and L. Waldmann, Z. Naturforsch. 25a, 336 (1970).

[16] H. Moraal, Z. Naturforsch. 28a, 824 (1973).

[17] F. M. Chen, H. Moraal and R. F. Snider, J. Chem. Phys. 57, 542 (1972).

[18] R. F. Snider, Physica 78, 387 (1974).

[19] W. E. Köhler, Z. Naturforsch. 30a, 117 (1975).

[20] W. K. Liu, F. R. McCourt, and W. E. Köhler, J. Chem. Phys. 71, 2566 (1979)

[21] W. E. Köhler, Z. Naturforsch. 29a, 1705 (1974).

[22] H. Moraal and R. F. Snider, Chem. Phys. Letters 9, 401 (1971).

[23] S. Chapman and T. G. Cowling, The Mathematical Theory of Non-Uniform Gases, Cambridge University Press, Cambridge 1964.

[24] L. S. Rodberg and R. M. Thaler, Introduction to the Quantum Theory of Scattering, Academic Press, New York 1967.

[25] A. R. Edmonds, Drehimpulse in der Quantenmechanik, Bibl. Inst. Mannheim 1964.

[26] S. Hess and L. Waldmann, Z. Naturforsch. 21 a, 1529 (1966).

[27] W. E. Köhler and H. F. P. Knaap, Z. Naturforsch. $31 \mathrm{a}, 1485$ (1976).

[28] W. E. Köhler, H. F. P. Knaap, G. E. J. Eggermont, and G. W.'t Hooft, Z. Naturforsch. 33a, 761 (1978).

[29] J. O. Hirschfelder, C. F. Curtiss, and R. B. Bird, Molecular Theory of Gases and Liquids, John Wiley, New York 1954.

[30] W. E. Köhler, Z. Naturforsch. 28a, 815 (1973). 